Pacific Journal of Mathematics

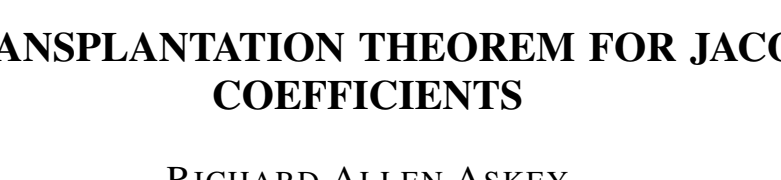




\section{A TRANSPLANTATION THEOREM FOR JACOBI COEFFICIENTS}

\section{RICHARD ASKEY}

Let $f(\theta)$ be integrable on $(0, \pi)$ and define

$$
a_{n}^{\alpha, \beta}=t_{n}^{\alpha, \beta} \int_{0}^{\pi} f(\theta) P_{n}^{(\alpha, \beta)}(\cos \theta)\left(\sin \frac{\theta}{2}\right)^{\alpha+(1 / 2)}\left(\cos \frac{\theta}{2}\right)^{\beta+(1 / 2)} d \theta
$$

where $P_{n}^{(\alpha, \beta)}(x)$ is the Jacobi polynomial of degree $n$, order $(\alpha, \beta)$ and

$$
\left[t_{n}^{\alpha, \beta}\right]^{2}=\frac{(2 n+\alpha+\beta+1) \Gamma(n+1) \Gamma(n+\alpha+\beta+1)}{\Gamma(n+\alpha+1) \Gamma(n+\beta+1)} .
$$

Then if $\alpha, \beta, \gamma, \delta \geqq-1 / 2$ we have

$$
\sum_{n=0}^{\infty}\left|a_{n}^{(r, \delta)}\right|^{p}(n+1)^{\sigma} \leqq A \sum_{n=0}^{\infty}\left|a_{n}^{(\alpha, \beta)}\right|^{p}(n+1)^{\sigma}
$$

for $1<p<\infty,-1<\sigma<p-1$ whenever the right hand side is finite.

From this result any norm inequality for Fourier coefficients can be transplanted to give a corresponding norm inequality for Fourier-Jacobi coefficients.

Let $P_{n}^{(\alpha, \beta)}(x)$ be defined by $(-1)^{n} 2^{n} n !(1-x)^{\alpha}(1+x)^{\beta} P_{n}^{(\alpha, \beta)}(x)=$ $(d / d x)^{n}\left\{(1-x)^{n+\alpha}(1+x)^{n+\beta}\right\}, \quad \alpha, \beta>-1$. The functions $P_{n}^{(\alpha, \beta)}(\cos \theta)$ are orthogonal on $(0, \pi)$ with respect to the measure

$$
\left(\sin \frac{\theta}{2}\right)^{2 \alpha+1}\left(\cos \frac{\theta}{2}\right)^{2 \beta+1} d \theta
$$

and

$$
\begin{aligned}
& \int_{0}^{\pi}\left[P_{n}^{(\alpha, \beta)}(\cos \theta)\right]^{2}\left(\sin \frac{\theta}{2}\right)^{2 \alpha+1}\left(\cos \frac{\theta}{2}\right)^{2 \beta+1} d \theta \\
& \quad=\frac{\Gamma(n+\alpha+1) \Gamma(n+\beta+1)}{(2 n+\alpha+\beta+1) \Gamma(n+\alpha+\beta+1) \Gamma(n+1)}=\left[t_{n}^{\alpha, \beta}\right]^{-2} .
\end{aligned}
$$

Observe that $t_{n}^{(\alpha, \beta)}=A n^{1 / 2}+O\left(n^{-1 / 2}\right)$ where $A$ is a constant whose numerical value is of no interest to us. For simplicity we set $R_{n}^{\alpha, \beta}(\theta)=$ $t_{n}^{\alpha, \beta} P_{n}^{(\alpha, \beta)}(\cos \theta)[\sin (\theta / 2)]^{\alpha+(1 / 2)}[\cos (\theta / 2)]^{\beta+(1 / 2)}$. The functions $\left\{R_{n}^{\alpha, \beta}(\theta)\right\}_{n=0}^{\infty}$ form a complete orthonormal sequence of functions on $(0, \pi)$. Also $R_{n}^{-1 / 2,-1 / 2}(\theta)=A \cos n \theta$ and $R_{n}^{1 / 2,1 / 2}(\theta)=A \sin (n+1) \theta$.

If $f(\theta) \in L^{1}(0, \pi)$ we define its Fourier-Jacobi coefficients by

$$
a_{n}^{\alpha, \beta}=\int_{0}^{\pi} f(\theta) R_{n}^{\alpha, \beta}(\theta) d \theta .
$$

We define $l^{p, \sigma}$ to be the space of sequences $\left\{a_{n}\right\}$ such that $\left\|a_{n}\right\|_{p, \sigma}=$ 
$\left[\sum_{n=0}^{\infty}\left|a_{n}\right|^{p}(n+1)^{\sigma}\right]^{1 / p}$ is finite. Our main theorem follows.

THEOREM 1. Let $\alpha, \beta, \gamma, \delta \geqq-1 / 2$ and $f(\theta) \in L^{1}(0, \pi)$. Let $a_{n}^{\alpha, \beta}$ and $a_{n}^{r, s}$ be defined by (2). Then if $1<p<\infty,-1<\sigma<p-1$ and if either $\left\|a_{n}^{\alpha, \beta}\right\|_{p, \sigma}$ or $\left\|a_{n}^{\gamma, \delta}\right\|_{p, \sigma}$ is finite so is the other and

$$
A \leqq\left\|a_{n}^{\alpha, \beta}\right\|_{p, \sigma} /\left\|a_{n}^{\gamma, \delta}\right\|_{p, \sigma} \leqq A
$$

where $A$ is independent of $f$ and thus of $\alpha_{n}^{\alpha, \beta}$ and $a_{n}^{\gamma, \delta}$.

For $\alpha=\beta, \gamma=\delta$ this theorem was proved in [1]. The last section of [1] gives two applications of this theorem. They can be carried over word for word to Jacobi coefficients. If all of the formulas for ultraspherical polynomials that were used in [1] were known for Jacobi polynomials, the proof of Theorem 1 could be exactly the same as the proof of the special case of it in [1]. While it is undoubtedly true that the relevant facts stated in [1] do generalize they are at present unknown. An example of such a fact is the following. Consider $P_{n}^{(\alpha, \beta)}(x) P_{m}^{(\alpha, \beta)}(x)$. This is a polynomial of degree $n+m$ and so

$$
P_{n}^{(\alpha, \beta)}(x) P_{m}^{(\alpha, \beta)}(x)=\sum_{k=0}^{n+m} \alpha_{k} P_{k}^{(\alpha, \beta)}(x) .
$$

If $\alpha \geqq \beta$ the conjecture is that $\alpha_{k} \geqq 0$. This is true for $\alpha=\beta$ and was used in [1]. The limiting result $\alpha \rightarrow \infty$ is also true and is stated in [4] as a result for Laguerre polynomials. For $\alpha=\beta+1$ it was proven in [6].

2. In this section we give various results that we need to prove Theorem 1.

For $0<\theta<\pi / 2, \alpha \geqq-1 / 2$, we have the following two inequalities

$$
\begin{gathered}
\left|P_{n}^{\alpha, \beta}(\cos \theta)\right|=O\left(n^{\alpha}\right), \\
\left|R_{n}^{\alpha, \beta}(\theta)\right|=\left|t_{n}^{\alpha, \beta}\left(\sin \frac{\theta}{2}\right)^{\alpha+(1 / 2)}\left(\cos \frac{\theta}{2}\right)^{\beta+(1 / 2)} P_{n}^{(\alpha, \beta)}(\cos \theta)\right| \leqq A .
\end{gathered}
$$

See $[7,(7.32 .6)]$. In (5) the power of $\cos \theta / 2$ can be changed at will since $\cos \theta / 2$ is bounded away from zero for $0 \leqq \theta \leqq \pi / 2$.

$$
\frac{d}{d x} P_{n}^{(\alpha, \beta)}(x)=\frac{1}{2}(n+\alpha+\beta+1) P_{n-1}^{(\alpha+1, \beta+1)}(x) .
$$

See $[7,(4.21 .7)]$.

The asymptotic formula we need is an easy consequence of two known results which we now state.

If $\alpha>-1, \beta$ real and $0<\theta \leqq \pi-\varepsilon, \varepsilon>0$, then 


$$
\begin{aligned}
& \left(\sin \frac{\theta}{2}\right)^{\alpha}\left(\cos \frac{\theta}{2}\right)^{\beta} P_{n}^{(\alpha, \beta)}(\cos \theta) \\
& \quad=N^{-\alpha} \frac{\Gamma(n+\alpha+1)}{\Gamma(n+1)}(\theta / \sin \theta)^{1 / 2} J_{\alpha}(N \theta)+R_{n}(\theta)
\end{aligned}
$$

where $N=n+(\alpha+\beta+1) / 2$ and

$$
R_{n}(\theta)= \begin{cases}\theta^{1 / 2} O\left(n^{-3 / 2}\right) & n^{-1} \leqq \theta \leqq \pi-\varepsilon \\ \theta^{\alpha+2} O\left(n^{\alpha}\right) & 0<\theta<n^{-1}\end{cases}
$$

$J_{\alpha}(x)$ is the Bessel function of the first kind of order $\alpha$. See [7, (8. 21.17)]. We also need a known asymptotic formula for $J_{\alpha}(x)$.

$$
\begin{aligned}
x^{1 / 2} J_{\alpha}(x)= & A \cos (x-\alpha \pi / 2-\pi / 4)\left[1+O\left(x^{-2}\right)\right] \\
& +A \sin (x-\alpha \pi / 2-\pi 4)\left[A x^{-1}+O\left(x^{-3}\right)\right], \quad x \rightarrow \infty .
\end{aligned}
$$

See $[7,(1.71 .8)]$. Combining (7), (8) and the asymptotic formula for $t_{n}^{\alpha, \beta}$ we get

$$
\begin{aligned}
R_{n}^{\alpha, \beta}(\theta)= & A \cos (N \theta-\alpha \pi / 2-\pi / 4)+A \sin (N \theta-\alpha \pi / 2-\pi / 4) /(N \theta) \\
& +O\left(N^{-1}\right)+O\left(N^{-2} \theta^{-2}\right), \quad 0<c / n \leqq \theta \leqq \pi / 2 .
\end{aligned}
$$

Finally we need a simple estimate for an integral.

$$
\int_{N}^{\infty} \frac{\cos y}{y} d y=O\left(N^{-1}\right), \quad N \rightarrow \infty .
$$

This follows on integrating by parts.

3. We assume that $f(\theta)$ is smooth enough, say $C^{2}$ and vanishing near 0 and $\pi$, so that the series $\sum a_{n} R_{n}^{\alpha, \beta}(\theta)$ converges uniformly on $[0, \pi]$. These conditions are sufficient for $a_{n}=O\left(n^{-2}\right)$, integrate by parts twice, and $\left|R_{n}^{\alpha, \beta}(\theta)\right| \leqq A$. We remove this condition after the following argument.

$$
a_{n}^{\gamma, \delta}=\int_{0}^{\pi} f(\theta) R_{n}^{\gamma, \delta}(\theta) d \theta=\sum_{k=0}^{\infty} a_{k}^{\alpha, \beta} \int_{0}^{\pi} R_{k}^{\alpha, \beta}(\theta) R_{n}^{\gamma, \delta}(\theta) d \theta=\sum_{k=0}^{\infty} a_{k}^{\alpha, \beta} R(k, n) .
$$

Since $P_{n}^{(\alpha, \beta)}(-x)=(-1)^{n} P_{n}^{(\beta, \alpha)}(x),[7,(4.1 .3)]$ it is sufficient to estimate $S(k, n)=\int_{0}^{\pi / 2} R_{k}^{\alpha, \beta}(\theta) R_{n}^{\gamma, \delta}(\theta) d \theta$. Also because we have made no assumptions about the relationships among $\alpha, \beta, \gamma, \delta$ it is sufficient to consider the case $k \geqq n$. We do this in two stages, $n \leqq k \leqq 2 n$ and $k \geqq 2 n$. For $n \leqq k \leqq 2 n$ the method is the same as in [1]. We repeat it here for convenience and because the other estimate is handled by a refinement of this argument. 


$$
S(k, n)=\int_{0}^{\pi / 2}=\int_{0}^{1 / k}+\int_{1 / k}^{\pi / 2}
$$

The first integral is $O\left(k^{-1}\right)$ since $R_{k}^{\alpha, \beta}(\theta)=O(1)$, see (5). In the second integral we use (9),

$$
\begin{aligned}
R_{n}^{\alpha, \beta}(\theta)= & A \cos \left(N \theta-\frac{\alpha \pi}{2}-\frac{\pi}{4}\right)+A \sin \left(N \theta-\frac{\alpha \pi}{2}-\frac{\pi}{4}\right) / N \theta \\
& +O\left(N^{-1}\right)+O\left(N^{-2} \theta^{-2}\right)
\end{aligned}
$$

to get

$$
\begin{aligned}
S(k, n) & =A \int_{1 / k}^{\pi / 2} \cos \left(K \theta-\frac{\alpha \pi}{2}-\frac{\pi}{4}\right) \cos \left(N \theta-\frac{\gamma \pi}{2}-\frac{\pi}{4}\right) d \theta \\
& +\frac{A}{K} \int_{1 / k}^{\pi / 2} \sin \left(K \theta-\frac{\alpha \pi}{2}-\frac{\pi}{4}\right) \cos \left(N \theta-\frac{\gamma \pi}{2}-\frac{\pi}{4}\right) \frac{d \theta}{\theta} \\
& +\frac{A}{N} \int_{1 / k}^{\pi / 2} \cos \left(K \theta-\frac{\alpha \pi}{2}-\frac{\pi}{4}\right) \sin \left(N \theta-\frac{\gamma \pi}{2}-\frac{\pi}{4}\right) \frac{d \theta}{\theta}+O\left(K^{-1}\right) .
\end{aligned}
$$

The first integral is $A /(K-N)+O\left(K^{-1}\right)$, the second is

$$
\frac{A}{K} \log \frac{K}{K-N}+O\left(K^{-1}\right),
$$

the third is $(A / N) \log N /(K-N)+O\left(K^{-1}\right)$ by a simple computation. The details are in [1]. The one time this argument breaks down is when $K=N$. In this case $S(k, n)=O(1)$ by (5).

Now we consider the case $k>2 n$. This time we need not be so careful, i.e., all our estimates may be $O$ estimates, but the details turn out to be harder than in the above case. This probably isn't necessary but we have not found a simple proof of the following estimates. There is one case, $\gamma=\alpha+2, \beta=\delta$, in which it is possible to give easy estimates as we will show later. But this is a very singular case.

As before $S(k, n)=\int_{0}^{1 / k}+\int_{1 / k}^{\pi / 2}$. The first integral is $O\left(K^{-1}\right)$ by (5). Next we show that in the second integral we may replace $R_{K}(\theta)$ by $\cos \{K \theta-(\alpha \pi / 2)-(\pi / 4)\}$. Using (9) we see it is sufficient to show that

$$
\begin{aligned}
& \frac{n^{1 / 2}}{K} \int_{1 / k}^{\pi / 2} \sin \left(K \theta-\frac{\alpha \pi}{2}-\frac{\pi}{4}\right) P_{n}^{(\gamma, \delta)}(\cos \theta)\left(\sin \frac{\theta}{2}\right)^{\gamma+(1 / 2)}\left(\cos \frac{\theta}{2}\right)^{\delta+(1 / 2)} \theta^{-1} d \theta \\
& =O\left(K^{-1}\right) .
\end{aligned}
$$

Integrating by parts and estimating we have 


$$
\begin{aligned}
\frac{n^{1 / 2}}{K^{2}} \int_{1 / k}^{\pi / 2} P_{n}^{(\gamma, \delta)}(\cos \theta)\left(\sin \frac{\theta}{2}\right)^{\gamma+(1 / 2)}\left(\cos \frac{\theta}{2}\right)^{\delta+(1 / 2)} \theta^{-1} d \cos \left(K \theta-\frac{\alpha \pi}{2}-\frac{\pi}{4}\right) \\
=\left.O\left(\frac{n^{1 / 2}}{K^{2}} P_{n}^{(\gamma, \delta)}(\cos \theta) \theta^{\gamma-(1 / 2)}\right)\right|_{1 / k} ^{\pi / 2}+O\left[\frac{n^{3 / 2}}{K^{2}} \int_{1 / k}^{\pi / 2} \cos \left(K \theta-\frac{\alpha \pi}{2}-\frac{\pi}{4}\right)\right. \\
\left.\cdot P_{n-1}^{(\gamma+1, \delta+1)}(\cos \theta)\left(\sin \frac{\theta}{2}\right)^{\gamma+(3 / 2)}\left(\cos \frac{\theta}{2}\right)^{\delta+(3 / 2)} \theta^{-1} d \theta\right] \\
\quad+O\left(\frac{n^{1 / 2}}{K^{2}} \int_{1 / k}^{\pi / 2} \cos \left(K \theta-\frac{\alpha \pi}{2}-\frac{\pi}{4}\right) P_{n}^{(\gamma, \delta)}(\cos \theta)\left(\sin \frac{\theta}{2}\right)^{\gamma-(1 / 2)}\right. \\
\left.\quad \cdot\left(\cos \frac{\theta}{2}\right)^{\delta+(3 / 2)} \theta^{-1} d \theta\right) \\
\quad+\operatorname{similar} \text { terms. }
\end{aligned}
$$

The integrated term is $O\left(K^{-1}\right)$ by (5). The second integral is

$$
O\left(K^{-2} \int_{1 / k}^{\pi / 2} \theta^{-2} d \theta\right)=O\left(K^{-1}\right)
$$

by (5). The first integral we write as $\int_{1 / k}^{1 / n}+\int_{1 / n}^{\pi / 2}$. Using (4) in $\int_{1 / k}^{1 / n}$ we have the bound $\left(n^{3 / 2} / K^{2}\right) \int_{1 / k}^{1 / n} n^{\gamma+1} \theta^{\gamma+(1 / 2)} d \theta=O\left(K^{-1}\right)$. In $\int_{1 / n}^{\pi / 2}$ we use (9) to get

$$
\begin{aligned}
& \frac{A n}{K^{2}} \int_{1 / n}^{\pi / 2} \cos \left(K \theta-\frac{\alpha \pi}{2}-\frac{\pi}{2}\right)\left[\cos \left(N \theta-\frac{\gamma \pi}{2}-\frac{\pi}{4}\right)\right. \\
& \left.\quad+\frac{A \sin \left(N \theta-\frac{\gamma \pi}{2}-\frac{\pi}{4}\right)}{N \theta}+O\left(N^{-1}\right)+O\left(N^{-2} \theta^{-2}\right)\right] \theta^{-1} d \theta \\
& =\frac{A n}{K^{2}} \int_{1 / n}^{\pi / 2} \frac{\cos (K-N) \theta}{\theta} d \theta+O\left(K^{-1}\right) \\
& \quad+\text { terms similar to the first. }
\end{aligned}
$$

Changing variables we get $\int_{(k-n) / n}^{(\pi / 2)(k-n)}(\cos y / y) d y$. Since $k>2 n$ we have $(k-n) / n \geqq 1$. Using (10) we get an estimate for the first term of the form $\left(A n / K^{2}\right)\{n /(K-n)\}=O\left(K^{-1}\right)$. Thus it is sufficient to consider

$$
t_{n}^{\gamma, \delta} \int_{1 / k}^{\pi / 2} \cos \left(K \theta-\frac{\alpha \pi}{2}-\frac{\pi}{4}\right) P_{n}^{(\gamma, \delta)}(\cos \theta)\left(\sin \frac{\theta}{2}\right)^{\gamma+(1 / 2)}\left(\cos \frac{\theta}{2}\right)^{\delta+(1 / 2)} d \theta .
$$

As above we break this integral into two parts $\int_{1 / k}^{1 / n}+\int_{1 / n}^{\pi / 2}$. We treat the first of these first. Integrating by parts and estimating we have 


$$
\begin{aligned}
\int_{1 / k}^{1 / n}= & O\left(K^{-1}\right)+\frac{t_{n}^{\gamma, \delta}}{K} \int_{1 / k}^{1 / n} \sin \left(K \theta-\frac{\alpha \pi}{2}-\frac{\pi}{4}\right) \frac{d}{d \theta}\left[P_{n}^{(\gamma, \delta)}(\cos \theta)\right. \\
& \left.\cdot\left(\sin \frac{\theta}{2}\right)^{\gamma+(1 / 2)}\left(\cos \frac{\theta}{2}\right)^{\delta+(1 / 2)}\right] d \theta .
\end{aligned}
$$

Using (6) and (4) we see that this integral is $O\left(K^{-1}\right)$.

In our one remaining integral we may use (9). However to get an estimate of the form $O\left(K^{-1}\right)$ we must first integrate by parts. Then we get

$$
\begin{aligned}
\frac{t_{n}^{\gamma, \delta}}{2 K}\left(\gamma+\frac{1}{2}\right) \int_{1 / n}^{\pi / 2} \sin \left(K \theta-\frac{\alpha \pi}{2}-\frac{\pi}{4}\right) P_{n}^{(\gamma, \delta)}(\cos \theta) \\
\cdot\left(\sin \frac{\theta}{2}\right)^{\gamma-(1 / 2)}\left(\cos \frac{\theta}{2}\right)^{\delta+(3 / 2)} d \theta \\
+\frac{t_{n}^{\gamma, \delta}}{K}[n+\gamma+\delta+1] \int_{1 / n}^{\pi / 2} \sin \left(K \theta-\frac{\alpha \pi}{2}-\frac{\pi}{4}\right) P_{n-1}^{(\gamma+1, \delta+1)}(\cos \theta) \\
\cdot\left(\sin \frac{\theta}{2}\right)^{\gamma+(3 / 2)}\left(\cos \frac{\theta}{2}\right)^{\delta+(3 / 2)} d \theta+O\left(K^{-1}\right)(\text { by }(5)) .
\end{aligned}
$$

For the first integral we have the estimate

$$
\begin{aligned}
& O\left[\frac { 1 } { K } \int _ { 1 / n } ^ { \pi / 2 } \operatorname { s i n } ( K \theta - \frac { \alpha \pi } { 2 } - \frac { \pi } { 4 } ) \left[\cos \left(N \theta-\frac{\gamma \pi}{2}-\frac{\pi}{4}\right)\right.\right. \\
& \left.\left.\quad+A(N \theta)^{-1} \sin \left(N \theta-\frac{\gamma \pi}{2}-\frac{\pi}{4}\right)+O\left(N^{-1}\right)+O\left(\theta^{-2} N^{-2}\right)\right]\left(\sin \frac{\theta}{2}\right)^{-1} d \theta\right] \\
& =O\left[\frac{1}{K} \int_{1 / n}^{\pi / 2} \frac{\sin (K-N) \theta}{\theta} d \theta\right]+O\left(K^{-1}\right)
\end{aligned}
$$

by (9) and the fact that $(1 / \sin \theta)-(1 / \theta)$ is bounded. As above this leads to the estimate $O\left(n / K^{2}\right)+O\left(K^{-1}\right)=O\left(K^{-1}\right)$.

A simple computation shows that $t_{n}^{\gamma, \delta}=A t_{n-1}^{\gamma+1, \delta+1}\left[1+O\left(n^{-1}\right)\right]$ so the second integral may also be estimated by using (9). The estimate is

$$
\begin{aligned}
O\left(\frac { n } { K } \int _ { 1 / n } ^ { \pi / 2 } \operatorname { s i n } ( K \theta - \frac { \gamma \pi } { 2 } - \frac { \pi } { 4 } ) \left[\cos \left(N \theta-\frac{\alpha \pi}{2}-\frac{\pi}{4}\right)\right.\right. \\
\left.\left.\quad+\frac{A \sin \left(N \theta-\frac{\alpha \pi}{2}-\frac{\pi}{4}\right)}{N \theta}+O\left(N^{-1}\right)+O\left(N^{-2} \theta^{-2}\right)\right] d \theta\right) \\
=O\left(\frac{n}{K} \int_{1 / n}^{\pi / 2} \sin (K-N) \theta d \theta\right)+O\left(\frac{n}{K} \int_{i / n}^{\pi / 2} \frac{\cos (K-N) \theta}{N \theta} d \theta\right) \\
\quad+\operatorname{similar} \text { terms }+O\left(K^{-1}\right) \\
=O\left(\frac{n}{K(K-N)}\right)+O\left(K^{-1}\right)=O\left(K^{-1}\right)
\end{aligned}
$$


by the same type of arguments that have been used often above. Combining all of the above estimates we see that

$$
\begin{aligned}
a_{n}^{\gamma, \delta}= & O\left[\frac{1}{n} \sum_{k=0}^{[n / 2]}\left|a_{k}^{\alpha, \beta}\right|\right]+A \sum_{k=[n / 2]}^{2 n} \frac{a_{k}^{\alpha, \beta}}{K-N} \\
& +\frac{A}{n} \sum_{k=[n / 2]}^{2 n} \alpha_{k}^{\alpha, \beta} \log \left|\frac{N}{K-N}\right|+A \sum_{k=[n / 2]}^{2 n} \frac{a_{k}^{\alpha, \beta}}{K} \log \left|\frac{K}{K-N}\right| \\
& +\left[\sum_{k=2 n}^{\infty} \frac{\left|a_{k}^{\alpha, \beta}\right|}{K}\right] .
\end{aligned}
$$

As in [1] all of the terms on the right are bounded operators in $l^{p, \sigma}$, $1<p<\infty,-1<\sigma<p-1$. Thus $\left\|a_{n}^{\gamma, \delta}\right\|_{p, \sigma} \leqq A\left\|a_{n}^{\alpha, \beta}\right\|_{p, \sigma}$ which is (3).

Let $g(\theta) \in C^{2}$ and vanish near 0 and $\pi$ and let $f(\theta) \in L^{1}(0, \pi)$. Define their Fourier-Jacobi coefficients, $b_{n}^{\alpha, \beta}$ and $\alpha_{n}^{\alpha, \beta}$ respectively, by (2). Then

$$
\sum_{n \equiv 0}^{\infty} b_{n}^{\gamma, \delta} a_{n}^{\gamma, \delta}=\int_{0}^{\pi} f(\theta) g(\theta) d \theta=\sum_{n=0}^{\infty} b_{n}^{\alpha, \beta} a_{n}^{\alpha, \beta}
$$

and thus $\{$ with $(1 / p)+(1 / q)=1\}$

$$
\begin{aligned}
\left\|a_{n}^{\gamma, \delta}\right\|_{p, \sigma} & =\left[\sum_{n=0}^{\infty}\left|a_{n}^{\gamma, \delta}\right|^{p}(n+1)^{\sigma}\right]^{1 / p}=\sup \sum a_{n}^{\gamma, \delta} b_{n}^{\gamma, \delta}=\sup \sum a_{n}^{\alpha, \beta} b_{n}^{\alpha, \beta} \\
& \leqq \sup \left\|a_{n}^{\alpha, \beta}\right\|_{p, \sigma}\left\|b_{n}^{\alpha, \beta}\right\|_{q, \sim q \sigma / p} \leqq A\left\|a_{n}^{\alpha, \beta}\right\|_{p, \sigma}
\end{aligned}
$$

by (3). Here the sup is taken over the sequences $b_{n}^{\gamma, \delta}$ with

$$
\sum\left|b_{n}^{\gamma, \delta}\right|^{q}(n+1)^{\sigma(1-q)} \leqq 1 .
$$

This completes the proof of Theorem 1.

There is a simple substitute in $l^{1}$ which follows easily from (11).

THEOREM 2. Let $\alpha, \beta, \gamma, \delta$ be as in Theorem 1 and assume $\sum\left|\alpha_{n}^{\alpha, \beta}\right| \log (n+2)<\infty$. Then $\sum\left|a_{n}^{\gamma, \delta}\right|<\infty$ where

$$
a_{n}^{\gamma, \delta}=\int_{0}^{\pi} f(\theta) R_{n}^{\gamma, \delta}(\theta) d \theta
$$

; $f(\theta)=\sum a_{n}^{\alpha, \beta} R_{n}^{\alpha, \beta}(\theta)$

The inequalities that are needed to prove Theorem 2 from (11) are in [3], where this result was proven for $\alpha=\beta=-1 / 2, \gamma=\delta=1 / 2$. To be pedantic here we must be careful for unless $\alpha=-1 / 2, R_{n}^{(\alpha, \beta)}(0)=0$ and so $f(\theta)=\sum a_{n}^{\alpha, \beta} R_{n}^{\alpha, \beta}(\theta)$ must vanish at $\theta=0$. Thus if $\alpha=-1 / 2$ we must assume $f(0)=0$ and similarly for $\beta=-1 / 2, \theta=\pi$. Theorem 2 is the one place where the above proof is an improvement over the proof in [1] (even in the case $\alpha=\beta, \gamma=\delta$ ) for using the proof in [1] we 
must add higher powers to the logarithm if $\alpha$ and $\gamma$ are far apart. Even this can be done away with if we use Theorem 4 which follows in the next section. However this problem would again arise if one tried to prove Theorem 2 for Jacobi (not ultraspherical) coefficients by the method in [1] by say holding $\beta$ fixed first and then varying it with fixed $\alpha$.

4. We conclude this paper with two simple theorems that hold in $l^{1}$. Since the details are easier we first give a theorem for Laguerre coefficients and then finally we give the corresponding theorem for Jacobi coefficients.

The Laguerre polynomial $L_{n}^{\alpha}(x)$ is defined by

$$
\sum_{n=0}^{\infty} L_{n}^{\alpha}(x) r^{n}=(1-r)^{-\alpha-1} \exp \left(\frac{-x r}{1-r}\right) .
$$

These functions satisfy

$$
\int_{0}^{\infty} L_{n}^{\alpha}(x) L_{m}^{\alpha}(x) x^{\alpha} e^{-x} d x=\frac{\Gamma(n+\alpha+1)}{\Gamma(n+1)} \delta_{n, m}, \quad \alpha>-1
$$

Let $f(x) \in L^{1}(0, \infty)$ and define its Fourier-Laguerre coefficient by

$$
a_{n}^{\alpha}=t_{n}^{\alpha} \int_{0}^{\infty} f(x) L_{n}^{\alpha}(x) x^{\alpha / 2} e^{-x / 2} d x .
$$

where

$$
t_{n}^{\alpha}=\left[\frac{\Gamma(n+1)}{\Gamma(n+\alpha+1)}\right]^{1 / 2} .
$$

We need one more fact about these functions.

$$
L_{n}^{\alpha+1}(x)=\sum_{j=0}^{n} L_{j}^{\alpha}(x)
$$

From this we see that

$$
L_{n}^{\alpha}(x)=L_{n}^{\alpha+1}(x)-L_{n-1}^{\alpha+1}(x) .
$$

Theorem 3. Let $f(x) \in L^{1}(0, \infty)$ and define $a_{n}^{\alpha}, a_{n}^{\alpha+2}$ by (13). Then if $\alpha>0$,

$$
A \leqq\left[\sum\left|a_{n}^{\alpha}\right|^{p}\right]^{1 / p} /\left[\sum\left|a_{n}^{\alpha+2}\right|^{p}\right]^{1 / p} \leqq A
$$

for $1 \leqq p<\infty$. If $-1<\alpha<0$ then (16) holds for $2 /(2+\alpha)<p<$ $-2 / \alpha$.

Using (12) and (13) we see that 


$$
\begin{aligned}
a_{n}^{\alpha+2} & =t_{n}^{\alpha+2} \int_{0}^{\infty} f(x) L_{n}^{\alpha+2}(x) x^{(\alpha / 2)+1} e^{-x / 2} d x \\
& =t_{n}^{\alpha+2} \sum_{k=0}^{\infty} t_{k}^{\alpha} a_{k}^{\alpha} \int_{0}^{\infty} L_{k}^{\alpha}(x) L_{n}^{\alpha+2}(x) x^{\alpha+1} e^{-x} d x \\
& =t_{n}^{\alpha+2} \sum_{k=0}^{n+1} t_{k}^{\alpha} a_{k}^{\alpha} \int_{0}^{\infty} L_{k}^{\alpha}(x) L_{n}^{\alpha+2}(x) x^{\alpha+1} e^{-x} d x
\end{aligned}
$$

Then using (14) and (15) we have

$$
\begin{aligned}
a_{n}^{\alpha+2}= & t_{n}^{\alpha+2} t_{0}^{\alpha} a_{0}^{\alpha} \int_{0}^{\infty} L_{0}^{\alpha}(x) \sum_{j=0}^{n} L_{j}^{\alpha+1}(x) x^{\alpha+1} e^{-x} d x \\
& +t_{n}^{\alpha+2} \sum_{k=1}^{n} t_{k}^{\alpha} a_{k}^{\alpha} \int_{0}^{\infty}\left[L_{k}^{\alpha+1}(x)-L_{k-1}^{\alpha+1}(x)\right]\left[\sum_{j=0}^{n} L_{j}^{\alpha+1}(x)\right] x^{\alpha+1} e^{-x} d x \\
& +t_{n}^{\alpha+2} t_{n+1}^{\alpha} a_{n+1}^{\alpha} \int_{0}^{\infty}-L_{n}^{\alpha+1}(x) L_{n}^{\alpha+1}(x) x^{\alpha+1} e^{-x} d x \\
= & t_{n}^{\alpha+2} t_{0}^{\alpha}\left[t_{0}^{\alpha+1}\right]^{-2} a_{0}^{\alpha}+t_{n}^{\alpha+2} \sum_{k=1}^{n} t_{k}^{\alpha} a_{k}^{\alpha}\left[\left(t_{k}^{\alpha+1}\right)^{-2}-\left(t_{k-1}^{\alpha+1}\right)^{-2}\right] \\
& -t_{n}^{\alpha+2} t_{n+1}^{\alpha}\left[t_{n}^{\alpha+1}\right]^{-2} a_{n+1}^{\alpha} .
\end{aligned}
$$

Thus

$$
\begin{aligned}
\left|a_{n}^{\alpha+2}\right| & \leqq A\left|a_{0}^{\alpha}\right| n^{-(\alpha / 2)-1}+A \sum_{k=1}^{n} n^{-(\alpha / 2)-1} k^{-\alpha / 2} k^{\alpha}\left|a_{k}^{\alpha}\right|+A\left|a_{n+1}^{\alpha}\right| \\
& \leqq A\left|a_{0}^{\alpha}\right|+A \sum_{k=1}^{n}\left|a_{k}^{\alpha}\right|(k / n)^{\alpha / 2} n^{-1}+A\left|a_{n+1}^{\alpha}\right|
\end{aligned}
$$

Similarly one can show that

$$
\left|a_{k}^{\alpha}\right| \leqq A\left|\alpha_{k-1}^{\alpha+2}\right|+A \sum_{n=k}^{\infty}\left|a_{n}^{\alpha+2}\right|(k / n)^{\alpha / 2} n^{-1} .
$$

Theorem 3 then follows from problem 346 in [5]. Actually there is one application of Theorem 3 and surprisingly it is for $\alpha$ negative. In a paper which will appear, Wainger and I prove the following theorem.

THEOREM A. Let $\alpha \geqq 0, f \in L^{1}(0, \infty)$ and define

$$
a_{n}^{\alpha}=\int_{0}^{\infty} f(x) t_{n}^{\alpha} L_{n}^{\alpha}(x) x^{\alpha / 2} e^{-x / 2} d x .
$$

Let $t(x)$ be a bounded function which is of bounded variation on $(0, \infty)$, with $\int_{0}^{\infty}|d t(x)| \leqq C$. Define

$$
T a_{n}^{\alpha}=\int_{0}^{\infty} t(x) f(x) t_{n}^{\alpha} L_{n}^{\alpha}(x) x^{\alpha / 2} e^{-x / 2} d x .
$$

Then this operator is bounded in $l^{p}, 4 / 3<p<4$, i.e. 


$$
\left[\sum\left|T a_{n}^{\alpha}\right|^{p}\right]^{1 / p} \leqq A C\left[\sum\left|a_{n}^{\alpha}\right|^{p}\right]^{1 / p}
$$

where $A$ is independent of $f(x)$ and of $t(x)$.

We used asymptotic estimates of Erdélyi which have only been proven for $\alpha \geqq 0$. See [2] where the dual result is proven. We can now extend this result to $\alpha \geqq-1 / 2$ by using Theorem 3 . Similar applications are given in [1] and we will not repeat the details here.

It would be interesting to extend Theorem 3 to get a theorem which corresponds to Theorem 1. The estimates of Erdélyi are probably not sufficient to allow one to prove this but they can probably be extended to give two terms plus an error and this might suffice.

The proof of the following theorem for Jacobi coefficients is exactly the same as the proof of Theorem 3 but the coefficients are not as simple so it looks more complicated.

THEOREM 4. Let $f(\theta) \in L^{1}(0, \pi)$ and define $a_{n}^{\alpha, \beta}, a_{n}^{\alpha+2, \beta}$ by (2). Then if $\alpha>-1 / 2, \beta>-1$, and if either $\sum\left|a_{n}^{\alpha, \beta}\right|$ or $\sum\left|\alpha_{n}^{\alpha+2, \beta}\right|$ converges so does the other and

$$
0<A \leqq \sum\left|\alpha_{n}^{\alpha, \beta}\right| / \sum\left|\alpha_{n}^{\alpha+2, \beta}\right| \leqq A<\infty
$$

with $A$ independent of $f$.

$$
\begin{aligned}
a_{n}^{\alpha+2, \beta}= & \int_{0}^{\pi} f(\theta) R_{n}^{\alpha+2, \beta}(\theta) d \theta=\sum_{k=0}^{\infty} \alpha_{k}^{\alpha, \beta} \int_{0}^{\pi} R_{n}^{\alpha+2, \beta}(\theta) R_{k}^{\alpha, \beta}(\theta) d \theta \\
= & t_{n}^{\alpha+2, \beta} \sum_{k=0}^{\infty} a_{k}^{\alpha, \beta} t_{k}^{\alpha, \beta} \int_{0}^{\pi} P_{n}^{(\alpha+2, \beta)}(\cos \theta) P_{k}^{(\alpha, \beta)}(\cos \theta) \\
& \cdot\left(\sin \frac{\theta}{2}\right)^{2 \alpha+3}\left(\cos \frac{\theta}{2}\right)^{2 \beta+1} d \theta \\
= & 2^{-\alpha-\beta-2} t_{n}^{\alpha+2, \beta} \sum_{k=0}^{\infty} a_{k}^{\alpha, \beta} t_{k}^{\alpha, \beta} \int_{-1}^{1} P_{n}^{(\alpha+2, \beta)}(x) P^{(\alpha, \beta)}(x)(1-x)^{\alpha+1}(1+x)^{\beta} d x \\
= & 2^{-\alpha-\beta-2} t_{n}^{\alpha+2, \beta} \sum_{k=0}^{n+1} a_{k}^{\alpha, \beta} t_{k}^{\alpha, \beta} R(k, n) .
\end{aligned}
$$

To estimate $R(k, n)$ we use the following.

$$
\begin{aligned}
& P_{n}^{(\alpha+1, \beta)}(x)= \frac{\Gamma(n+\beta+1)}{\Gamma(n+\alpha+\beta+2)} \\
& \cdot \sum_{j=0}^{n} \frac{(2 j+\alpha+\beta+1) \Gamma(j+\alpha+\beta+1)}{\Gamma(j+\beta+1)} P_{j}^{(\alpha, \beta)}(x), \\
& P_{n}^{(\alpha, \beta)}(x)=\frac{n+\alpha+\beta+1}{2 n+\alpha+\beta+1} P_{n}^{(\alpha+1, \beta)}(x) \\
& \quad-\frac{n+\beta}{2 n+\alpha+\beta+1} P_{n-1}^{(\alpha+1, \beta)}(x) .
\end{aligned}
$$


(18) follows from (17) and (17) is (4.5.3) in [7]. Using (17) and (18) we see that

$$
\begin{aligned}
R(k, n)= & \sum_{j=0}^{n} \frac{\Gamma(n+\beta+1)(2 j+\alpha+\beta+2) \Gamma(j+\alpha+\beta+2)}{\Gamma(n+\alpha+\beta+3) \Gamma(j+\beta+1)(2 k+\alpha+\beta+1)} \\
& \cdot \int_{-1}^{1} P_{j}^{(\alpha+1, \beta)}(x)\left[(k+\alpha+\beta+1) P_{k}^{(\alpha+1, \beta)}(x)\right. \\
& \left.-(k+\beta) P_{k-1}^{(\alpha+1, \beta)}(x)\right](1-x)^{\alpha+1}(1+x)^{\beta} d x .
\end{aligned}
$$

For $1 \leqq k \leqq n$ we have

$$
\begin{aligned}
R(k, n)= & \frac{\Gamma(n+\beta+1)}{\Gamma(n+\alpha+\beta+3)}\left[\frac{(2 k+\alpha+\beta+2) \Gamma(k+\alpha+\beta+2)}{\Gamma(k+\beta+1)(2 k+\alpha+\beta+1)}\right. \\
& \left.\cdot \frac{(k+\alpha+\beta+1)}{\left[t_{k}^{\alpha+1, \beta}\right]^{2}}-\frac{(2 k+\alpha+\beta) \Gamma(k+\alpha+\beta+1)(k+\beta)}{\Gamma(k+\beta)(2 k+\alpha+\beta+1)\left[t_{k-1}^{\alpha+1, \beta}\right]^{2}}\right] \\
= & \frac{\Gamma(n+\beta+1)}{\Gamma(n+\alpha+\beta+3)(2 k+\alpha+\beta+1)} \\
& \cdot\left[\frac{\Gamma(k+\alpha+2)(k+\alpha+\beta+1)}{\Gamma(k+1)}-\frac{\Gamma(k+\alpha+1)(k+\beta)}{\Gamma(k)}\right] \\
= & \frac{\Gamma(n+\beta+1) \Gamma(k+\alpha+1)}{\Gamma(n+\alpha+\beta+3)(2 k+\alpha+\beta+1) \Gamma(k+1)} \\
& \cdot[(k+\alpha+1)(k+\alpha+\beta+1)-k(k+\beta)] \\
= & O\left(n^{-\alpha-2} k^{\alpha}\right) .
\end{aligned}
$$

For $k=0, R(k, n)=O\left(n^{-\alpha-2}\right)$ follows easily from (17), (18) and (1). For $k=n+1, R(k, n)=O\left(n^{-1}\right)$ also follows easily from these same formulas. Thus we have

$$
\left|a_{n}^{\alpha+2, \beta}\right| \leqq A n^{-1} \sum_{k=0}^{n+1}\left|\alpha_{k}^{\alpha, \beta}\right|(k / n)^{\alpha+1 / 2}
$$

and

$$
\sum_{n=0}^{\infty}\left|a_{n}^{\alpha+2, \beta}\right| \leqq A \sum_{n=0}^{\infty}\left|a_{n}^{\alpha, \beta}\right|
$$

follows easily by interchanging the order of summation. The other inequality follows by the same argument.

\section{REFERENCES}

1. R. Askey and S. Wainger, A transplantation theorem for ultraspherical coefficients, Pacific J. Math. 16 (1966), 393-405.

2. Mean convergence of expansions in Laguerre and Hermite series, Amer. J. Math. 87 (1965), 695-708.

3. R. P. Boas, Absolute convergence and integrability of trigonometric series, J. Rat. Mech. Anal. 5 (1956), 621-632. 
4. A. Erdélyi, On some expansions in Laguerre polynomials, J. Lond. Math. Soc. 13 (1938), 154-156.

5. G. H. Hardy, J. E. Littlewood, and G. Polya, Inequalities, Cambridge, 1952.

6. E. Hylleraas, Linearization of products of Jacobi polynomials, Math. Scand. 10 (1962), 189-200.

7. G. Szegö, Orthogonal Polynomials, Amer. Math. Soc. Coll. Pub. Vol. 23, revised edition (1959), New York.

Received April 1966. Supported in part by NSF grant GP-3483.

THE UNIVERSITY OF WISCONSIN

MADISON, WISCONSIN 


\section{PACIFIC JOURNAL OF MATHEMATICS}

\section{EDITORS}

\section{H. SAMELSON}

Stanford University

Stanford, California

\author{
J. P. JANS \\ University of Washington \\ Seattle, Washington 98105
}

\section{J. DuGUNDJI}

University of Southern California

Los Angeles, California 90007

RichaRd ARENS

University of California

Los Angeles, California 90024

\section{ASSOCIATE EDITORS}

E. F. BECKENBACH
B. H. NeUManN

F. WOLF

K. YOSIDA

\section{SUPPORTING INSTITUTIONS}

UNIVERSITY OF BRITISH COLUMBIA

CALIFORNIA INSTITUTE OF TECHNOLOGY

UNIVERSITY OF CALIFORNIA

MONTANA STATE UNIVERSITY

UNIVERSITY OF NEVADA

NEW MEXICO STATE UNIVERSITY

OREGON STATE UNIVERSITY

UNIVERSITY OF OREGON

OSAKA UNIVERSITY

UNIVERSITY OF SOUTHERN CALIFORNIA
STANFORD UNIVERSITY

UNIVERSITY OF TOKYO

UNIVERSITY OF UTAH

WASHINGTON STATE UNIVERSITY

UNIVERSITY OF WASHINGTON

AMERICAN MATHEMATICAL SOCIETY

CHEVRON RESEARCH CORPORATION

TRW SYSTEMS

NAVAL ORDNANCE TEST STATION

Mathematical papers intended for publication in the Pacific Journal of Mathematics should be typewritten (double spaced). The first paragraph or two must be capable of being used separately as a synopsis of the entire paper. It should not contain references to the bibliography. Manuscripts may be sent to any one of the four editors. All other communications to the editors should be addressed to the managing editor, Richard Arens at the University of California, Los Angeles, California 90024.

50 reprints per author of each article are furnished free of charge; additional copies may be obtained at cost in multiples of 50 .

The Pacific Journal of Mathematics is published monthly. Effective with Volume 16 the price per volume ( 3 numbers) is $\$ 8.00$; single issues, $\$ 3.00$. Special price for current issues to individual faculty members of supporting institutions and to individual members of the American Mathematical Society: $\$ 4.00$ per volume; single issues $\$ 1.50$. Back numbers are available.

Subscriptions, orders for back numbers, and changes of address should be sent to Pacific Journal of Mathematics, 103 Highland Boulevard, Berkeley 8, California.

Printed at Kokusai Bunken Insatsusha (International Academic Printing Co., Ltd.), No. 6, 2-chome, Fujimi-cho, Chiyoda-ku, Tokyo, Japan.

PUBLISHED BY PACIFIC JOURNAL OF MATHEMATICS, A NON-PROFIT CORPORATION

The Supporting Institutions listed above contribute to the cost of publication of this Journal, but they are not owners or publishers and have no responsibility for its content or policies. 


\section{Pacific Journal of Mathematics}

\section{Vol. 21, No. $3 \quad$ BadMonth, 1967}

Richard Allen Askey, A transplantation theorem for Jacobi coefficients . . . 393

Raymond Balbes, Projective and injective distributive lattices .......... 405

Raymond Balbes and Alfred Horn, Order sums of distributive lattices . . . . 421

Donald Charles Benson, Nonconstant locally recurrent functions ........ 437

Allen Richard Bernstein, Invariant subspaces of polynomially compact operators on Banach space ............................... 445

Robert F. Brown, Fixed points and fibre ................... 465

David Geoffrey Cantor, On the Stone-Weierstrass approximation theorem for valued fields ................................ 473

James Walton England, Stability in topological dynamics .............. 479

Alessandro Figà-Talamanca and Daniel Rider, A theorem on random

Fourier series on noncommutative groups.................. 487

Sav Roman Harasymiv, A note of dilations in $L^{p} \ldots \ldots \ldots \ldots \ldots \ldots . \ldots 493$

J. G. Kalbfleisch, A uniqueness theorem for edge-chromatic graphs ....... 503

Richard Paul Kelisky and Theodore Joseph Rivlin, Iterates of Bernstein polynomials .................................... 511

D. G. Larman, On the union of two starshaped sets ............... 521

Henry B. Mann, Josephine Mitchell and Lowell Schoenfeld, Properties of differential forms in $n$ real variables ...................... 525

John W. Moon and Leo Moser, Generating oriented graphs by means of team comparisons .

Veikko Nevanlinna, A refinement of Selberg's asymptotic equation ...

Ulrich Oberst, Relative satellites and derived functors of functors with additive domain ..............................

John Vincent Ryff, On Muirhead's theorem...............

Carroll O. Wilde and Klaus G. Witz, Invariant means and the Stone-Čech compactification 